\title{
Quantitative phase and polarization imaging through an optical fiber applied to detection of early esophageal tumorigenesis
}

George S. D. Gordon

James Joseph

Maria P. Alcolea

Travis Sawyer

Calum Williams

Catherine R. M. Fitzpatrick

Philip H. Jones

Massimiliano di Pietro

Rebecca C. Fitzgerald

Timothy D. Wilkinson

Sarah E. Bohndiek 


\title{
Quantitative phase and polarization imaging through an optical fiber applied to detection of early esophageal tumorigenesis
}

\author{
George S. D. Gordon, ${ }^{a}$ James Joseph, ${ }^{b, c}$ Maria P. Alcolea, ${ }^{d}$ Travis Sawyer, ${ }^{b, c}$ Calum Williams, ${ }^{a, b}$ \\ Catherine R. M. Fitzpatrick, ${ }^{a}$ Philip H. Jones, ${ }^{e}$ Massimiliano di Pietro, ${ }^{e}$ Rebecca C. Fitzgerald, ${ }^{e}$ \\ Timothy D. Wilkinson, ${ }^{a}$ and Sarah E. Bohndiek ${ }^{b, c, *}$ \\ aUniversity of Cambridge, Department of Engineering, Cambridge, United Kingdom \\ bUniversity of Cambridge, Department of Physics, Cavendish Laboratory, Cambridge, United Kingdom \\ ${ }^{\circ}$ Cancer Research UK Cambridge Institute, Li Ka Shing Centre, Cambridge, United Kingdom \\ dUniversity of Cambridge, Wellcome Trust MRC Stem Cell Institute, Cambridge, United Kingdom \\ eUniversity of Cambridge, MRC Cancer Unit, Hutchison/MRC Research Centre, Cambridge, United Kingdom
}

\begin{abstract}
Phase and polarization of coherent light are highly perturbed by interaction with microstructural changes in premalignant tissue, holding promise for label-free detection of early tumors in endoscopically accessible tissues such as the gastrointestinal tract. Flexible optical multicore fiber (MCF) bundles used in conventional diagnostic endoscopy and endomicroscopy scramble phase and polarization, restricting clinicians instead to low-contrast amplitude-only imaging. We apply a transmission matrix characterization approach to produce full-field en-face images of amplitude, quantitative phase, and resolved polarimetric properties through an MCF. We first demonstrate imaging and quantification of biologically relevant amounts of optical scattering and birefringence in tissue-mimicking phantoms. We present an entropy metric that enables imaging of phase heterogeneity, indicative of disordered tissue microstructure associated with early tumors. Finally, we demonstrate that the spatial distribution of phase and polarization information enables label-free visualization of early tumors in esophageal mouse tissues, which are not identifiable using conventional amplitude-only information. (c) The Authors. Published by SPIE under a Creative Commons Attribution 4.0 Unported License. Distribution or reproduction of this work in whole or in part requires full attribution of the original publication, including its DOI. [DOI: 10.1117/1.JBO.24.12.126004]
\end{abstract}

Keywords: optical fibers; quantitative phase imaging; polarimetry; cancer.

Paper 190292R received Aug. 30, 2019; accepted for publication Nov. 18, 2019; published online Dec. 16, 2019.

\section{Introduction}

White-light endoscopy is the standard-of-care for inspecting large areas of the gastrointestinal (GI) tract and lung for premalignant change (dysplasia) and cancer. ${ }^{1}$ For example, Barrett's esophagus is an acquired metaplastic condition that predisposes patients to the development of esophageal adenocarcinoma. The cancer risk for Barrett's patients increases significantly in the presence of premalignant transformation (dysplasia), up to more than $30 \%$ per year. ${ }^{1}$ Early identification of dysplasia enables curative intervention through simple endoscopic resection or radiofrequency ablation. ${ }^{2}$ Unfortunately, the current surveillance procedure uses white-light endoscopy combined with random biopsy, which together show only $40 \%$ to $64 \%$ sensitivity for dysplasia, leading to high miss rates. ${ }^{3}$ The 5 -year survival rate for esophageal cancer is only $15 \%$, yet can be as high as $80 \%$ when patients are diagnosed with early-stage disease, ${ }^{3}$ hence improvements in endoscopic early detection methodologies are urgently needed. While application of dyes can improve contrast, ${ }^{4}$ their use lengthens procedure times and can lead to toxicities $;{ }^{5}$ label-free approaches could better address the clinical unmet need for improved contrast of dysplastic tissue.

Endoscopic imaging of the esophagus can be performed in two modes: (1) the conventional "red-flag" imaging, which provides a wide field-of-view of the whole lumen to identify regions of suspected dysplasia and (2) emerging "optical

*Address all correspondence to Sarah E. Bohndiek, E-mail: seb53@cam.ac.uk biopsy," which aims to confirm the presence of dysplasia in a suspect region to better direct tissue biopsies or ultimately avoid the need for physical biopsy. ${ }^{6,7}$ In either mode, label-free imaging must interrogate an endogenous mechanism of contrast between healthy and diseased tissues. In addition to highdefinition white-light endoscopy, autofluorescence and narrowband imaging are label-free modalities often applied for red-flag imaging in upper GI endoscopy. Although they offer improved performance over the current standard-of-care, white-light imaging, they show limited specificity for detecting dysplasia. ${ }^{8,9}$ Fluorescence lifetime imaging has been proposed for endoscopic red-flag imaging but typically requires costly pulsed laser sources and gated detectors. ${ }^{10}$

A number of label-free optical biopsy modalities have shown promise to improve identification of dysplasia, including optical coherence tomography (OCT), ${ }^{11,12}$ angle-resolved lowcoherence intereferometry, ${ }^{13}$ elastic scattering spectroscopy, ${ }^{14}$ and Raman spectroscopy. ${ }^{15}$ While many red-flag approaches now employ "chip-on-tip" cameras, optical biopsy is usually achieved with the introduction of an optical fiber endomicroscope through the accessory channel of the red-flag endoscope. As these modalities only interrogate a single sample point or narrow field-of-view, they must be applied following red-flag imaging using another modality. Additionally, these label-free modalities typically require costly light sources (e.g., swept wavelength or supercontinuum sources for $\mathrm{OCT}^{16}$ ) and detectors (e.g., sensitive spectrometers for Raman and interferometric spectrometers for OCT). Hence there remains an unmet need 
for a label-free modality that offers rich contrast with relatively low-cost instrumentation and could offer both red-flag and optical biopsy analysis.

Localized proliferation of cells in dysplasia scatters light. ${ }^{1}$ This scattering can be completely characterized by measuring the reflection matrix, for example, by angular scanning microscopy. ${ }^{17}$ However, in many cases, phase imaging is sufficient to identify increased scattering by measuring abnormally distorted wavefronts. ${ }^{18}$ Phase imaging has also been demonstrated in thick biological samples, which are the most relevant for clinical applications. ${ }^{19}$ Polarimetric imaging measurements of diattenuation, retardance, and circularity can also be modified by scattering, as well as by the higher concentrations of optically anisotropic molecules, such as collagen, abundant in tumors. ${ }^{20}$ Light scattering spectroscopy has shown promise for detecting dysplasia using phase and polarization information but interrogates only a narrow field-of-view; ${ }^{14}$ this limitation is partially mitigated by OCT, which uses lateral scanning mechanisms and can be integrated into capsule endoscopes, ${ }^{14}$ but interpretation of the resulting cross-sectional images remains challenging. ${ }^{16}$ Adding phase or polarization sensitivity to chip-on-tip distal sensors is also difficult, as standard cameras do not capture this information; specialist cameras for the purpose are expensive and not easily miniaturized. ${ }^{21,22}$ Furthermore, these approaches typically require dedicated complex instrumentation, which has limited endoscopic application. Phase imaging through rigid endoscopes has recently been demonstrated, ${ }^{23}$ but flexible endoscopes are required for imaging inside the GI tract.

Exploiting clinically approved flexible multicore fibers (MCFs) to relay optical information from within the patient to the imaging system outside would be advantageous to enable direct, wide-field, en-face phase and polarization imaging with comparatively simple and low-cost elements. ${ }^{24,25}$ Unfortunately, MCFs inherently scramble phase and polarization information due to bending- and temperature-induced variations in glass refractive index, limiting diagnostic potential. Unscrambling these properties could, however, be achieved by measuring and inverting the fiber transmission matrix (TM), a complex linear mapping between the two fiber facets. ${ }^{26}$ Applying a recently reported ${ }^{27} \mathrm{TM}$ characterization architecture for MCFs, we show that quantitative phase- and polarization-resolved images can be obtained in transmission mode from tissue-mimicking phantoms that, respectively, contain physiologically relevant concentrations of optical scatterers and birefringent materials. We quantify scattering by presenting a spatial entropy metric and show that this accurately reflects reduced scattering coefficients of the prepared phantoms. We then perform a feasibility study to assess the potential of extracting these parameters to provide contrast within a tissue context. To achieve this, we apply the MCF as a "holographic endomicroscope," noting that it also has the potential to perform red-flag imaging because of the adjustable working distance. ${ }^{27}$ We use this to demonstrate label-free visualization of early tumors within healthy esophageal tissue taken from a mouse model of early disease. The demonstrated feasibility of the transmission-mode imaging presented here represents a motivating step toward development of a reflectionmode system that could be translated for in vivo use.

\section{Methods}

\subsection{Holographic Endoscopy}

We exploited a novel TM characterization architecture to enable wide-field imaging of quantitative phase- and polarizationresolved (i.e., holographic) properties of biological samples through a flexible MCF bundle (FIGH-06-350G, Fujikura; length of $2 \mathrm{~m}, 6000$ cores, core diameter of $\sim 2.9 \mu \mathrm{m}$, core spacing of $4.4 \mu \mathrm{m}$, and outer diameter of $350 \pm 20 \mu \mathrm{m}$ ). Figure 1 shows a schematic of the system, which is described in detail elsewhere. ${ }^{27}$ Briefly, data recording for holographic imaging through the fiber is performed in two stages: (1) characterizing the TM of the fiber and (2) recording, via the fiber, amplitude and phase images in two polarizations of the sample.

In the fiber characterization stage, a sequence of predetermined fields (an array of spots) is translated across the distal facet of the MCF using a holographic spatial mode and polarization state generator (described in Ref. 27), and the resultant amplitude and phase are imaged in two polarizations. The use

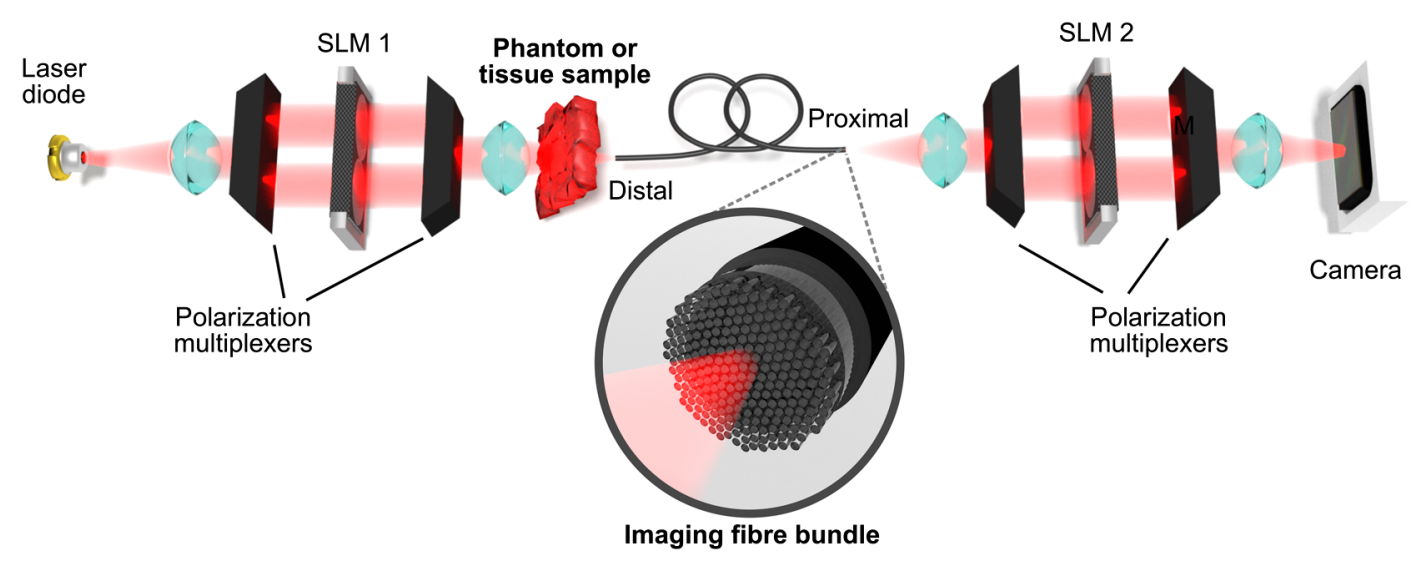

Fig. 1 Schematic of experimental setup for characterization of an MCF and recording of phase and polarization images of tissue samples and phantoms. Spatial light modulators (SLMs) are used with polarization multiplexers (comprising polarizing beam splitters and waveplates, see Ref. 27) to create polarization-diverse sample illumination and polarization-diverse imaging through an MCF. SLM1 illuminates the phantom or tissue sample with a programmable optical field and generates reference fields for fiber characterization, whereas SLM2 enables imaging of amplitude, phase, and polarization of light exiting the fiber bundle. ${ }^{27}$ 
of an array of spots exploits the sparse structure of the MCF TM to enable highly parallelized TM characterization measurements resulting in a significant speed-up (12-fold here because 12 spots are used) that is independent of MCF size.

In the image recording stage, the sample (e.g., tissue) is placed at the distal facet of the fiber. At a working distance of $0 \mathrm{~mm}$, imaging is performed through a square subsection of the MCF giving a field of view of $200 \times 200 \mu \mathrm{m}$, representing $2 / 3$ of the maximum imaging area. The remaining fiberlets carry a stable phase reference. In this mode, the imaging system is akin to an endomicroscope, but since the working distance can be electronically controlled, ${ }^{27}$ the field of view could in principle be extended with potential to be a red-flag device.

A broad, Gaussian illumination is then projected onto the sample and the light exiting the other side travels through the fiber and is then recorded, thus performing transmission-mode imaging. The illumination is swept through several different elliptical polarization states. Samples larger than the field of view of the MCF are translated to multiple positions using a stage.

Using this raw data, the TM can be recovered, the image of the sample can be reconstructed and, finally, biologically relevant optical parameters can be extracted. For polarimetric imaging, Jones calculus is applicable here because the light is temporally and spatially coherent due to the laser diode and the single-mode filtering of the cores, respectively, thus depolarization is negligible. ${ }^{28}$ The total acquisition time for an amplitude, phase, and polarization image set is $8.3 \mathrm{~s}$, and the time taken to fully characterize the fiber is $50.8 \mathrm{~min}$. However, by modifying our setup to use of state-of-the-art TM characterization techniques, we estimate these times could be reduced to $<0.1$ and 22 s, respectively. ${ }^{27,29}$

\subsection{Image Data Recovery and Analysis}

\subsubsection{Transmission matrix calculation}

Before image reconstruction can begin, the dual-polarization MCF TM must first be recovered using the data recorded in the fiber characterization stage (Sec. 2.1). This is done by considering corresponding pairs of input and output fields. Input and output fields are arranged into vectors and then concatenated, respectively, to form a matrix of inputs, $\mathbf{X}$, and a matrix of outputs, $\mathbf{Y}$, which are related by the TM, $\mathbf{A}$. The problem is then cast as a linear inverse problem and each row of $\mathbf{A}^{-1}, \mathbf{a}$, can be solved using the corresponding row of $\mathbf{X}, \mathbf{x}$ :

$\min \|\mathbf{a}\|_{1}$ subject to $\left\|\mathbf{x}^{T}-\mathbf{Y}_{\text {sub }}^{T} \mathbf{a}^{T}\right\|_{2}<\delta$.

This L1-norm minimization represents a convex optimization $^{30}$ and is solved using the SPGL1 package. ${ }^{31}$ This approach further exploits sparsity as is described in detail in Ref. 27. In this way, the inverse TM can be constructed row by row.

TM recovery currently takes around an hour but could be reduced to $<1$ min by replacing the current iterative approach ( $>1000$ Fourier transforms required) with transport-of-intensity equation methods (two Fourier transforms and one derivative required). ${ }^{32}$ Furthermore, frameworks that avoid the need for explicit $\mathrm{TM}$ reconstruction and directly reconstruct the image data have already been reported. ${ }^{33}$ Image recovery itself takes $<0.1 \mathrm{~s}$.

\subsubsection{Retrieval of quantitative phase- and polarization-resolved images}

Next, the inverse TM is applied to measured raw fields exiting the MCF. In general multiscattering media, the eigenvalues of the TM are distributed according to a quarter-circle law and so such inversion introduces noise in the eigenmodes with lower eigenvalues. ${ }^{34-36}$ However, as the optical fibers used here are made of low-loss glass and are designed to guide light, the eigenvalue distribution approaches uniformity, provided the number of modes interrogated is less than the modal "cutoff." ${ }^{, 36,37}$ For this particular imaging configuration and fiber, we have previously measured the TM condition number to be $<2.5$ under a range of bending configurations, which allows for high-fidelity TM inversion. ${ }^{38}$

Following TM inversion, we can accurately reconstruct the amplitude and phase in two polarizations, i.e., a full optical field, with a resolution of $9.0 \pm 2.6 \mu \mathrm{m} .{ }^{27}$ If the sample was translated, the relevant images are stitched together. If a longer working distance from the distal facet was used, the focus is adjusted computationally using a Fresnel transform. ${ }^{27}$

The recovered images inherently contain quantitative phase information. However, to extract interpretable polarimetric parameters, we first use the multiple illumination elliptical polarization states (Sec. 2.1) to determine the Jones matrix at each pixel. We then use a Bayesian inference approach to fit a model comprising an elliptical retarder followed by a partial polarizer, which represents a matrix factorization. This then produces a full set of polarimetric parameters. ${ }^{27}$

\subsubsection{Calculating entropy and expectation}

To identify biological lesions in otherwise healthy tissue using quantitative phase and polarization images, we apply two image filters: (1) spatial entropy, denoted as $H(.$.$) and (2) spatial$ expectation, denoted as $E(.$.$) . These can both be determined$ under a unified framework that estimates the probability distribution of spatial points within a filtering window. The size of the filter window is chosen to be 15 because it is sufficiently narrow so as not to "smooth out" the smallest lesions ( $\sim 30$ pixels here) but offers sufficient data points to reliably fit a two-dimensional (2-D) distribution. This then provides the differential entropy of the inferred distribution as $H(.$.$) and$ the mean as $E(.$.$) , effectively two spatial filters with a common$ length scale.

The distribution fitting process is detailed in Fig. 2. We assume here that pixels in this region are independently distributed. Correlation between pixels can be accounted for by computing joint histograms, e.g., using a gray-level co-occurrence matrix, ${ }^{39}$ but empirically this approach has a negligible impact on resultant entropy maps.

To achieve practical computation speeds, fitting is performed by evaluating predetermined analytic expressions for maximum likelihood parameters of chosen distributions.

The distribution parameters extracted from fitting, mean, and variance are used to determine the "differential entropy" metric, which is derived from the Kullback-Leibler divergence and thus measures how uniformly distributed the values are. ${ }^{40}$ Computed over a spatial region, this gives a measure of spatial heterogeneity indicative of disordered tissue microstructure in tumors. This heterogeneity can then be exploited as an effective contrast mechanism, which has been validated in reflection-mode imaging of tumors using spatial frequency-domain imaging. ${ }^{41}$ 


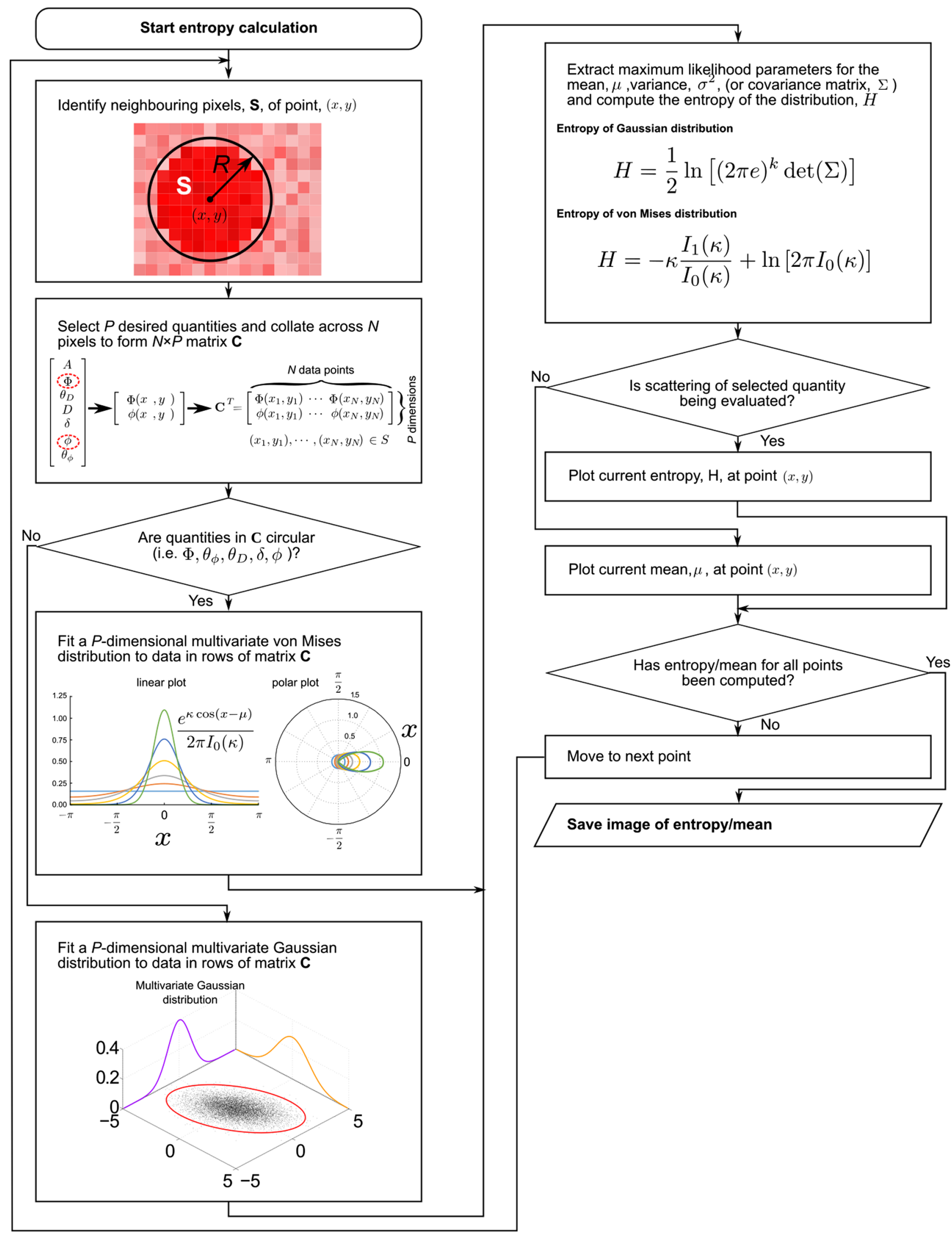

Fig. 2 The distribution fitting algorithm used to compute entropy and expectation metrics from images of amplitude, phase, and polarization. The value of $R$, the size of the filter window, is chosen to be 15 because it is sufficiently narrow so as not to smooth out the smallest lesions ( 30 pixels in size in this dataset) but offers sufficient data points to reliably fit a 2-D distribution. 
From the fitted distribution at each pixel, $(x, y)$, we compute the differential entropy, denoted as $H[g(x, y)]$ where $g()$ may be an amplitude or phase value or a polarimetric property.

When applied to quantitative phase images, this entropy metric gives a good indication of scattering-induced heterogeneity. However, when imaging phase, lower power pixels result in higher uncertainty in phase and therefore higher entropy, introducing a power dependence. To isolate the specific influence of the scattering properties of the sample on entropy, we define scattering-induced entropy as

$H_{\text {scat }}(x, y)=\frac{H\left[\beta_{\text {dist }}(x, y)\right]-H_{\text {pow }}\left[A^{2}(x, y)\right]}{H_{\text {pow }}\left[A^{2}(x, y)\right]}$,

where $H[.$.$] is the entropy function, \beta_{\text {dist }}(x, y)$ is the parameter of the distribution fitted to the phase of pixels neighboring $(x, y)$, $H_{\text {pow }}$ is the power-loss-induced entropy determined experimentally using a series of neutral density filters, and $A^{2}(x, y)$ is the average power level for the neighboring pixels of $(x, y)$.

By computing entropy for a sliding window over the entire raw image, we produce an "entropy filtered" image of the desired quantity. Entropy filtering has been successfully applied in the field of amplitude-only texture analysis for classification of diseased tissues. ${ }^{42-44}$ If instead of plotting entropy, we plot the mean of the fitted distributions at each spatial point, this gives images of the spatial expectation, $E$, effectively an averaging filtering on the same length scale as entropy. The use of analytical equations for entropy and expectation combined with efficient GPU parallelization means that entropy and expectation filters take $<0.1 \mathrm{~s}$ to act on raw data.

\subsection{Preparing Tissue-Mimicking Phantoms}

The sensitivity of the holographic endomicroscope to biologically relevant concentrations of scattering and birefringence through phase and polarization imaging was evaluated using tissue-mimicking phantoms. Optically scattering phantoms $(n=2)^{45}$ were prepared by adding varying volumes of prewarmed intralipid (20\% emulsion, I141, Sigma-Aldrich) to liquid $1.5 \mathrm{w} / \mathrm{v} \%$ agar solutions (05039, Fluka) to provide reduced scattering coefficients in the range from 0.125 to $2 \mathrm{~cm}^{-1}$. A $0.75 \%$ volume of $0.5 \mathrm{mg} \mathrm{ml}^{-1}$ nigrosin (198285, Sigma-Aldrich) was also added to provide an absorption coefficient of $0.005 \mathrm{~mm}^{-1}$. Birefringent phantoms $(n=4)$ were prepared using acrylamide/bis-acrylamide, 30\% solution (A3699, Sigma-Aldrich) catalyzed using tetramethylethylenediamine (TEMED, T9281, Sigma-Aldrich) and ammonium persulfate (APS, A3678, Sigma-Aldrich). Phantoms were fabricated inside a ventilated fumehood cupboard by mixing $30 \mathrm{ml}$ of acrylamide/ bis-acrylamide with $600 \mu \mathrm{l}$ of APS and $40 \mu \mathrm{l}$ of TEMED under thorough vortexing. For both scattering and birefringent phantoms, the liquid solution was poured into petri dishes (CELLSTAR 628-160, Greiner Bio-One) and then allowed to set at room temperature.

A double integrating sphere (DIS) system $^{46}$ was used to determine the reduced scattering and absorption coefficients of the phantoms. The DIS setup uses two highly reflective spheres (Labsphere), with Lambertian surfaces made from polytetrafluoroethylene, to capture and quantify the amount of transmitted and reflected light. A broadband tungsten halogen lamp (AvaLight-HAL-MINI, Avantes) and two fiber-optic spectrometers (AvaSpec-ULS2048, Avantes) served as the light source and detectors, respectively. The inverse-adding doubling algorithm was used to compute the optical properties of the phantoms from the reflectance, transmittance, and reference measurements. ${ }^{47}$ The scattering-induced entropy is used as the contrast metric when evaluating the holographic endomicroscopy performance.

\subsection{Imaging Biological Samples}

To test the ability of the holographic endomicroscope to identify tissue abnormalities in a label-free manner, we used ex vivo samples of mouse esophagus from healthy untreated controls $(n=3$; 7 healthy areas analyzed) and carcinogen-treated animals $(n=6$; 13 distinct lesions analyzed). The esophagus is an ideal model for validating this technology because it is endoscopically accessible, exhibits well-defined multistage carcinogenesis that can be tracked, and has multiple stages of disease represented in space. Samples consisted of a thin layer of esophageal epithelium, providing a representative model of surface scattering and polarimetric effects in the first few superficial cell layers where esophageal tumors are usually detected by endoscopy. All experiments were approved by the University of Cambridge local ethical review committees and conducted according to Home Office project licenses P14FED054 and 70/8866. Experimental mice were doubly transgenic for the inducible Cre allele AhcreERT and the conditional reporter allele EYFP targeted to the Rosa 26 locus (AhcreERTR26flEYFP/wt). ${ }^{48}$ Strains were maintained in a C57B16 background. Experiments were carried out with male and female animals. No gender-specific differences were observed.

Esophageal tumors were induced using a chemical carcinogen derived from cigarette smoke (diethylnitrosamine, SigmaAldrich). The carcinogen was administered at a concentration of $0.04 \mathrm{mg} \mathrm{l}^{-1}$ in sweetened drinking water to induce sporadic mutations. Adult animals (over 10-weeks old) were treated 3 days a week for 8 weeks as previously described. ${ }^{48}$ After carcinogen treatment, animals were aged from 6 to 9 months. Controls represent untreated animals. For epithelial tissue preparation, the esophagus was cut open longitudinally, dissected into rectangular pieces of $\sim 5 \mathrm{~mm} \times 8 \mathrm{~mm}$, and incubated for 2 to $3 \mathrm{~h}$ in $5 \mathrm{mM}$ EDTA at $37^{\circ} \mathrm{C}$. The epithelium was then carefully peeled away from underlying connective tissue with fine forceps. The tissue was fixed in $4 \%$ paraformaldehyde in phosphate-buffered saline (PBS) for $30 \mathrm{~min}$. For immunofluorescence staining, wholemounts were blocked for $1 \mathrm{~h}$ in staining buffer $(0.5 \%$ bovine serum albumin, $0.25 \%$ fish skin gelatin, and $0.5 \%$ triton X-100 in PBS) with $10 \%$ donkey or goat serum, according to the secondary antibody of choice. Antibodies for keratin 14 (Covance; PRB-155P; 1/1000) and keratin 4 (Vector; VP-C399; 1/1000) were incubated overnight in staining buffer, followed by washing for $2 \mathrm{~h}$ with $0.2 \%$ Tween-20 in PBS. Samples finally underwent overnight staining with $1 \mu \mathrm{g} \mathrm{ml}^{-1}$ 4',6-diamidino-2-phenylindole (DAPI). For imaging, the esophageal epithelium was laid flat on a glass side and a coverslip was placed on top and sealed. These samples are sufficiently thin that they have very high-power transmission and the power-loss-induced entropy is negligible so that $H_{\text {scat }}(x, y) \approx H\left[\beta_{\text {dist }}(x, y)\right] / k$, where $k=H_{\text {pow }}\left[A^{2}(x, y)\right]$ is a scale factor approximately constant across all samples and spatial positions. Therefore, $H\left[\beta_{\text {dist }}(x, y)\right]$ is used as the contrast metric for tissue samples.

To identify healthy regions and those containing early abnormalities or definite lesions, reference measurements were taken 
using bright-field, phase-contrast, and polarization microscopy (BX51-P, Olympus), as well as confocal fluorescence microscopy (Leica TCS SP5 II system: optimal pinhole; speed $400 \mathrm{~Hz}$; line average 3; resolution $1024 \times 1024$; image reconstruction using Volocity 6, PerkinElmer). An expert observer (M.A.) examined the confocal fluorescence microscopy images to confirm that no cells were present under the keratin 14 positive epithelial basal layer and to identify abnormal areas and lesions in the issue. The expert observer had previously validated the fluorescence staining approach for identification of abnormal areas and lesions in this mouse model using genetic analyses. ${ }^{48} \mathrm{~A}$ semiautomated registration process was then applied. The microscopy images were registered using the edges of the tissue samples as corresponding points between the samples; a $2 \times 2$ transformation matrix was then found and singular value decomposition was used to obtain scale and rotation. A similar process was then applied to find the transformation between the phase-contrast microscopy image and the stitched endoscopic phase image, using striations in the tissue as corresponding points.

\subsubsection{Contrast-to-noise ratio calculation}

The utility of endoscopically measured amplitude, phase, and polarimetric properties for detecting early tumors in the mouse samples is evaluated by calculating the contrast-to-noise ratio (CNR) between lesions and healthy tissue for each parameter, $p$, as

$$
\mathrm{CNR}=\frac{\mu\left[F\left(p_{\text {lesion }}\right)\right]-\mu\left[F\left(p_{\text {healthy }}\right)\right]}{\sigma\left[F\left(p_{\text {healthy }}\right)\right]},
$$

where $F(.$.$) represents either entropy, H(.$.$) , or expectation,$ $E(.),. \mu[.$.$] represents the mean of a quantity, and \sigma[.$.$] represents$ the standard deviation of a quantity. As discussed previously, the spatial expectation filter, $E(.$.$) , averages over the same spatial$ area as the entropy filter, $H(.$.$) , to ensure resolution of features$ is comparable.

For each CNR value computed, the lesion values are taken from the specific tissue sample tested (aggregated across all lesions if there are multiple), but the healthy values are aggregated from across all nine mouse samples. Because it accounts for different noise levels, CNR can be used to compare the results obtained using fluorescence, amplitude, phase, and polarization measurements. This metric is computed for the DAPI images as a reference, then for the amplitude entropy, phase entropy, retardance expectation, retardance axis expectation, diattenuation expectation, and diattenuation axis expectation and compared using a paired two-tailed $t$-test.

\subsubsection{Receiver operating characteristic calculation}

For the mouse tissue samples, the receiver operating characteristic curve is computed for a quantity $Q$ (e.g., fluorescence expectation and phase entropy) by applying a threshold, $t_{Q}$, to each pixel, $p$, in the data sets $L$, containing all pixels situated in a lesion region across all samples, and $R$, containing all pixels situated in a healthy region across all samples. This produces thresholded data sets defined as

$$
\begin{aligned}
& L_{T}(p)=\left\{\begin{array}{ll}
0, & L(p) \leq t_{Q} \\
1, & L(p)>t_{Q}
\end{array}\right. \text { and } \\
& R_{T}(p)= \begin{cases}1, & R(p) \leq t_{Q} \\
0, & R(p)>t_{Q}\end{cases}
\end{aligned}
$$

Sensitivity and specificity are then computed, respectively, as

$$
\frac{\sum_{P} L_{T}(p)}{\left|L_{T}\right|} \text { and } \frac{\sum_{P} R_{T}(p)}{\left|R_{T}\right|},
$$

where $|L|$ indicates the cardinality (i.e., number of elements) in the set $L$. The receiver operating characteristic (ROC) curve is produced by varying $t_{Q}$ between the minimum and maximum values across all data points (i.e., the extrema of set $L \cup R$ ).

\section{Results}

\subsection{Evaluation of Biologically Relevant Variations in Phase and Polarization}

Tissue-mimicking phantoms were used to emulate the expected scattering-induced phase variation and polarization effects of diseased tissue. For scattering, we imaged phantoms made of polyacrylamide gel mixed with varying concentrations of intralipid. The raw phase images clearly show a more perturbed wavefront resulting from scattering [Fig. 3(a)]. Spatial scattering-induced phase entropy increases significantly with intralipid scatterer concentration [Fig. 3(b)].

For birefringence, we produced transparent phantoms using polyacrylamide gel, which has a strain-dependent birefringence. The birefringent phantoms were mounted on a custom-fabricated mechanical stretcher [Fig. 4(a)] to create a strain-induced birefringence over the endomicroscopic fieldof-view. Deformation contours across the birefringent phantoms mounted in the stretcher were assessed by drawing a grid pattern onto the polyacrylamide gel using a marker pen. Qualitative observation of the contours after stretching revealed a macroscopic nonlinearity in the deformation. However, over the small field-of-view of the endomicroscope, the stretch was approximately linear. Due to the constant volume of the material, the change in the thickness upon stretching must be accounted for when plotting retardance, by considering the change in area between the two clamps and dividing the measured retardance by the inferred width. A clear change in retardance can be seen in the raw images and histograms [Fig. 4(b)], and a significant positive trend is observed as strain is increased [Fig. 4(c), $\left.r^{2}=0.71\right]$. Other polarization properties did not change significantly.

\subsection{Imaging Early Tumors in Mouse Esophagus}

We then sought to test the potential of our quantitative phaseand polarization-resolved imaging to detect early lesions of the esophagus. We imaged samples of mouse esophagus containing abnormal tissue consisting of early preneoplastic tumors $(n=6$, 13 distinct lesions) and control tissues ( $n=3,7$ healthy areas analyzed). Samples consisted of a thin layer of esophageal epithelium, providing a representative model of surface scattering and polarimetric effects in the first few superficial cell layers. As for the phantom study, we used a spatial phase entropy filter, denoted $H(\phi)$, to quantify scattering. The same filter was applied to the amplitude images to produce amplitude entropy, 


\section{(a)

Phase
(radians)
Raw phase entropy (a.u.)}

(b)
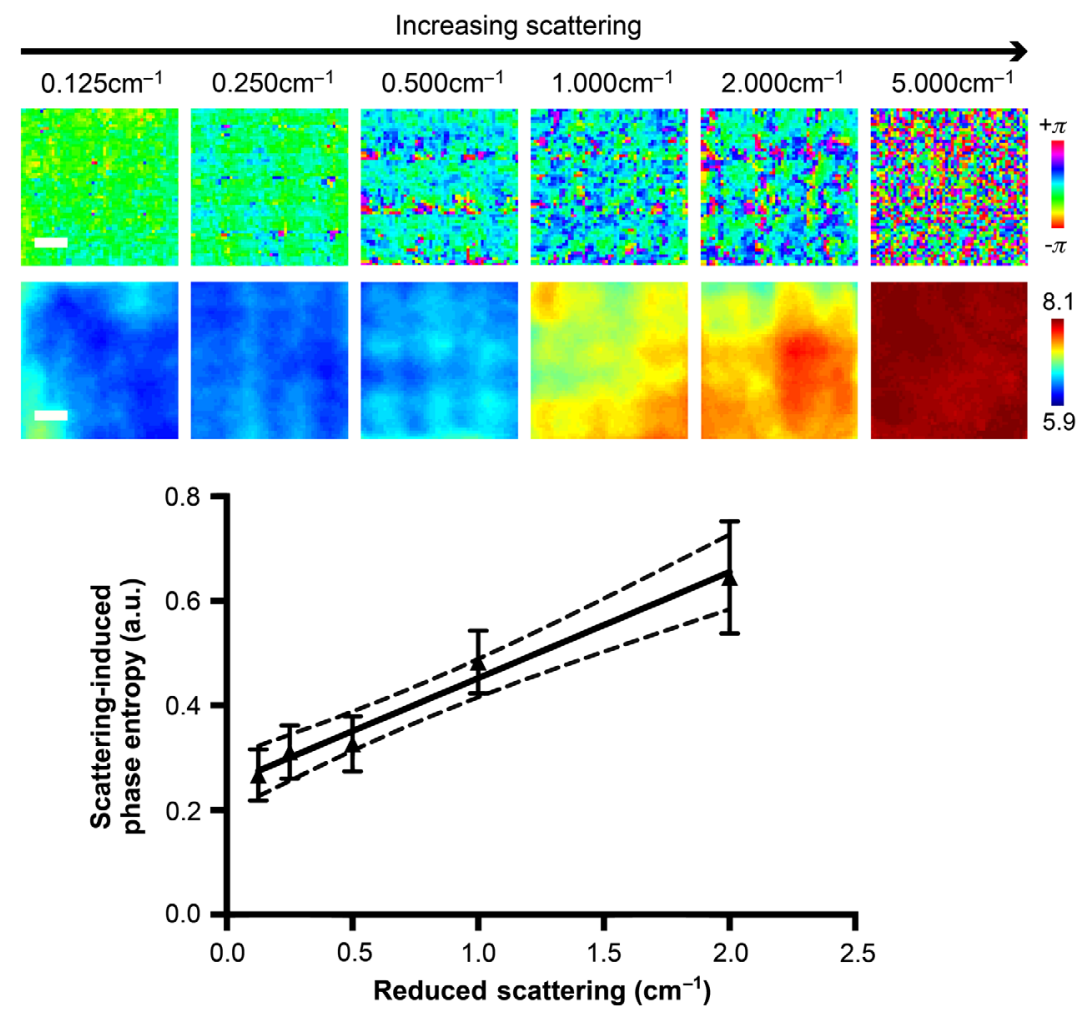

Fig. 3 Biologically relevant variations in phase can be detected. (a) Raw phase images enable the scattering of light due to intralipid to be directly observed, whereas phase entropy images produce quantified scattering maps. (b) Scattering-induced entropy shows a significant linear relationship $\left[y=(0.20 \pm 0.02) x+(0.25 \pm 0.02), r^{2}=0.9816, p=0.001 ; n=2\right]$ with increased intralipid scattering concentration. Valid measurements are bounded by the effect of power-loss-induced entropy, and for the last two samples, this is the dominant effect and so they are excluded from the trend. Image scale bar: $200 \mu \mathrm{m}$.

$H(A)$, as well as to all polarimetric images. Additionally, a spatial averaging filter, denoted $E(p)$, is applied to the raw amplitude, phase, and polarimetric images so that the presented spatial resolution is the same as the entropy filtered images allowing comparison between the metrics.

An example of the full range of imaging parameters available from the holographic endomicroscope for one full sample is illustrated in Fig. 5. Reference images were acquired using bright-field, fluorescence, phase-contrast, and crossed-polarization microscopy [Fig. 5(a)]. The lesion is not visible in the bright-field microscope but is apparent in phase-contrast and cross-polarization microscopy, indicating as expected that phase and polarization can provide information additional to amplitude-only imaging. This is in agreement with our hypothesis that phase and polarization properties of a sample can be measured with high fidelity through an MCF when using a TM recovery approach. The contrast observed in the cross-polarized microscope images [e.g., Fig. 5(a)] suggests polarization effects are present in the lesion but not in healthy tissue. The crossed-polarized microscope cannot resolve whether the underlying cause of this change in polarization is due to birefringence or diattenuation. We therefore consider the biological origins of polarization effects in early tumors-increasing anisotropic tissue microstructure and dense birefringent collagen networks arising during tumor growth-and consider jointly the polarimetric properties that might be affected, $\theta_{D}, \theta_{\phi}$, and $\phi .{ }^{49}$ Contrast of these individual parameters may vary between samples due to, for example, sample orientation, so by combining them (a simple sum is used here) we produce a more broadly defined "polarimetric contrast."

In some of the images, rectangular artifacts are observed, arising from stitching together multiple fields of view. Though these contribute noise, the statistical significance of our method is still sufficiently high to distinguish lesion tissue, and they could be reduced in the future by compensating for nonuniform illumination (e.g., speckle averaging illumination ${ }^{50}$ ).

The raw information obtained from the holographic endomicroscope [Fig. 5(b)] are not informative, but processing to display localized expectation and entropy provides some interesting observations [Figs. 5(c) and 5(d)]. Phase entropy shows high signal in the lesion area but is also sensitive to scattering caused by a tissue fold (verified in the fluorescence image) and the sample edge. The polarization metric of diattenuation axis $\left(\theta_{D}\right)$ also shows a strong signal at the position of the lesion.

Image regions of interest containing lesions or healthy regions from all tissue samples investigated are illustrated in Fig. 6 for the subset of holographic endomicroscope parameters that appeared to be qualitatively more sensitive to the presence of lesions during the full sample analyses. These are compared to fluorescence microscopy data, which is only available after applying a nuclear stain to the samples to highlight areas of abnormal tissue architecture and increased cell density routinely used to identify early tumors. ${ }^{48}$ We provide fluorescence microscopy data here as a gold-standard reference, rather than as an endoscopic comparator. Several label-free quantities measured 
(a)
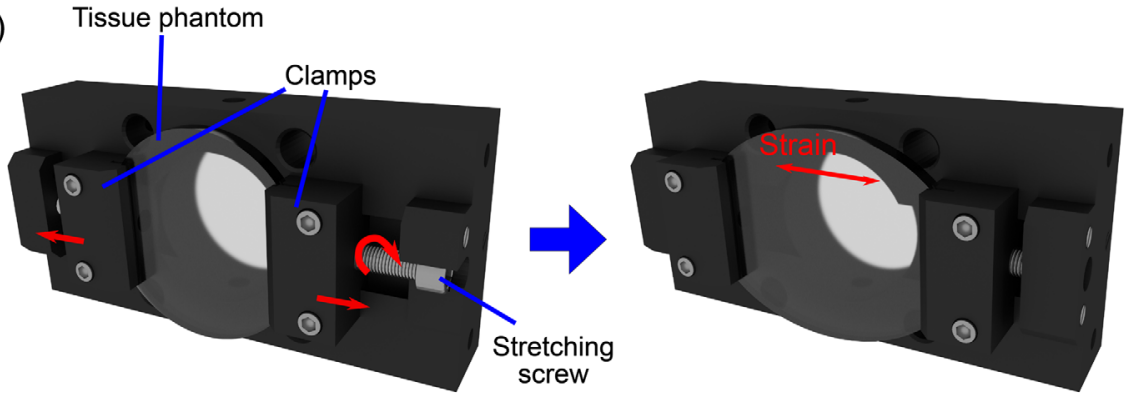

(b)

(badians)
(rance
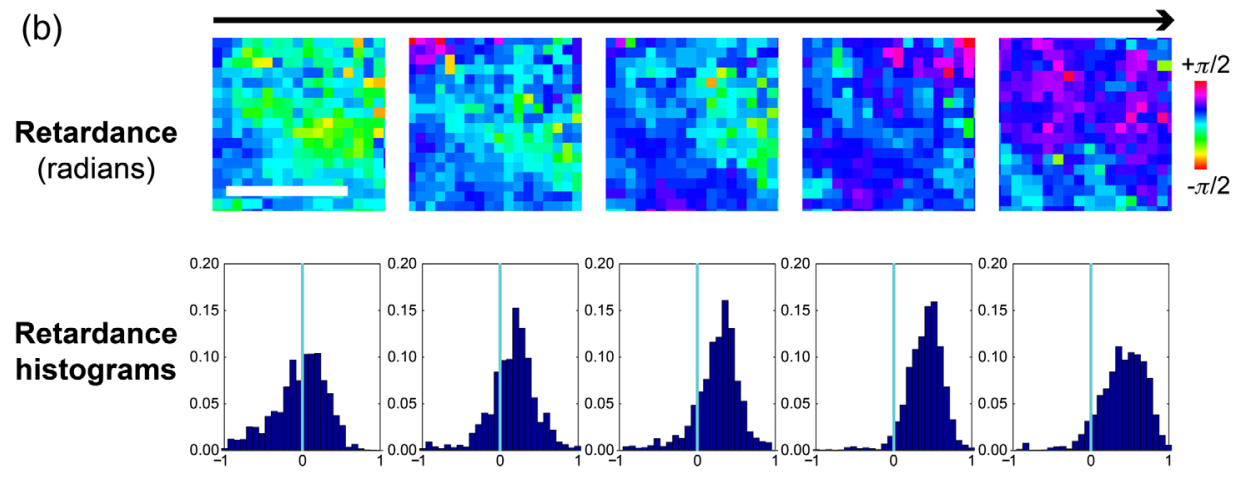

(c)

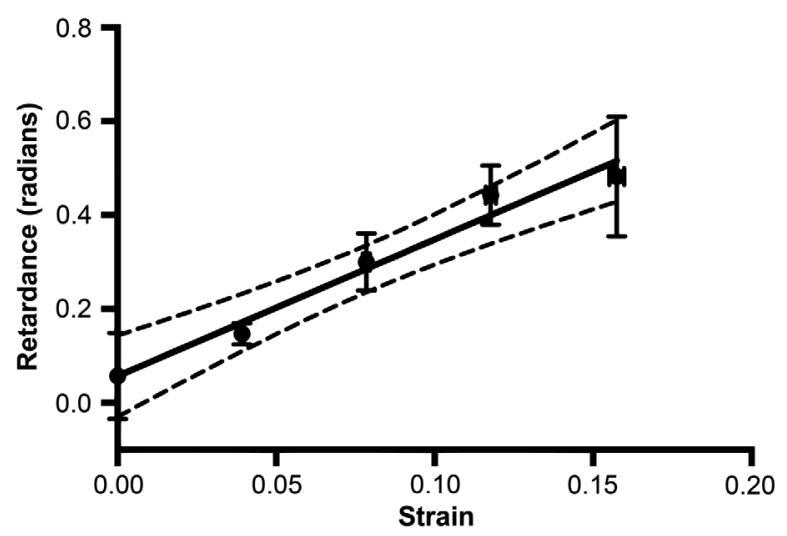

Fig. 4 Biologically relevant variations in polarization can be detected. (a) Schematic of the phantom stretcher assembly used for mounting and stretching birefringent phantoms. The phantom is held in place by the clamps, which are securely fastened with screws. The stretching screws (one on either side) are turned in uniform increments to stretch the phantom by increasing amounts in the lateral axis. (b) Raw images showing recovered retardance as a function of increasing strain with associated histograms. (c) Retardance increases significantly as a function of strain $[y=(2.91 \pm 0.29) x+(0.06 \pm 0.03)$, $\left.r^{2}=0.9725, p=0.002 ; n=4\right]$. Image scale bar: $200 \mu \mathrm{m}$.

with our holographic endomicroscope, particularly phase entropy, provide consistently high signal in the lesion areas.

Quantitative analysis was then performed using these images. CNR (defined in Sec. 2.4.1) was computed separately for each of the six abnormal tissue samples to evaluate the potential of the holographic endomicroscope parameters to identify lesions within a given tissue sample [Fig. 7(a)]. Sample edges were excluded from the analysis, but folds were included. Phase entropy was found to provide significantly improved CNR compared to amplitude entropy ( $p=0.0014)$, being comparable to the CNR obtained using the fluorescence nuclear stain $(p=0.150)$ but in this case acquired in a label-free manner. Individual polarimetric properties do not show significant increase over amplitude entropy. However, if we sum together properties that share a common biological origin $\left(\theta_{D}, \theta_{\phi}\right.$, and $\phi$ as above ${ }^{49}$ ) their contrast accumulates. The resultant metric is significantly better than amplitude entropy $(p=0.034)$ and again comparable to fluorescence imaging $(p=0.160)$. It should be noted that the high variance observed within these CNR calculations is caused by inherent biological variation rather than technical limitation, as CNR was calculated separately for each sample.

Finally, we applied a binary classifier to produce an ROC curve, showing the sensitivity and specificity across the sample set [Fig. 7(b)]. While the sample size is limited, encouragingly the phase entropy metric performs significantly better than amplitude entropy (similar to the existing standard-of-care) and lies close to the fluorescence reference, indicating superior performance for this label-free metric compared to conventional amplitude-only imaging or fluorescence-based approaches that require the application of dyes. The combined polarization metric performs worse with binary classification than amplitude 
(a)

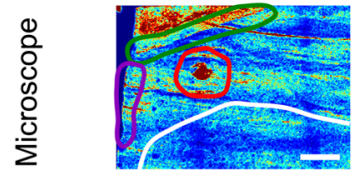

Fluorescence

(b)

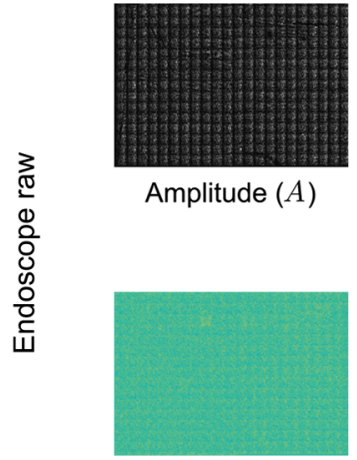

Diattenuation $(D)$

(c)

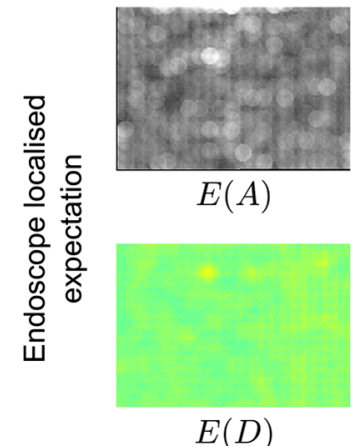

(d)

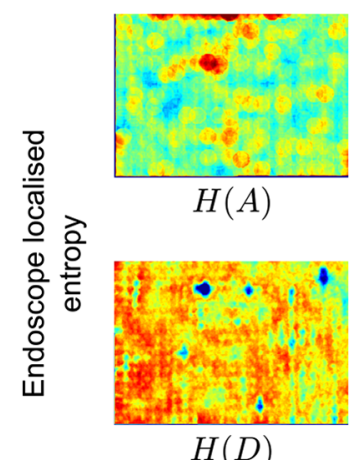

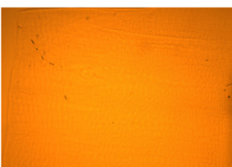

Brightfield

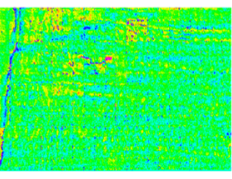

Phase $(\Phi)$

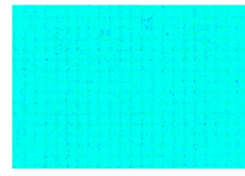

Retarder circularity $(\delta)$

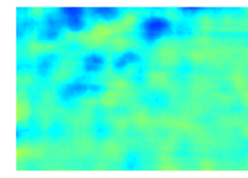

$E(\Phi)$

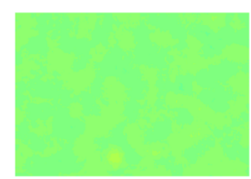

$E(\delta)$
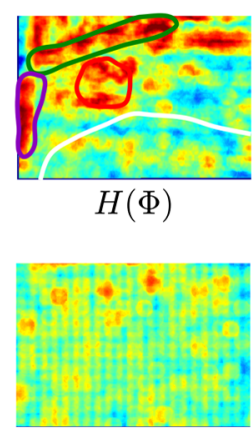

$H(\delta)$

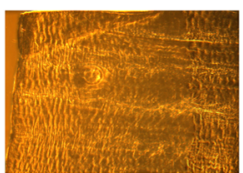

Phase contrast

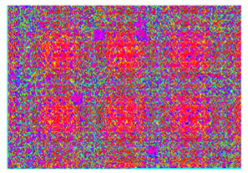

Retardance axis orientation $\left(\theta_{\phi}\right)$

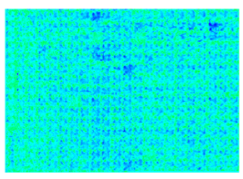

Retardance $(\phi)$

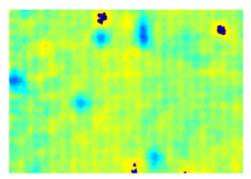

$E\left(\theta_{\phi}\right)$

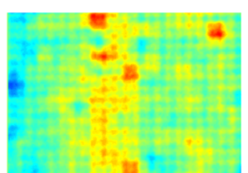

$E(\phi)$

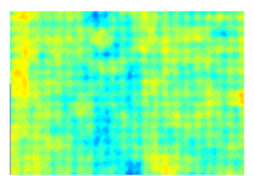

$H\left(\theta_{\phi}\right)$

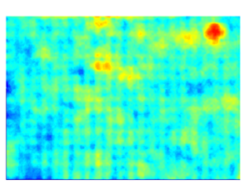

$H(\phi)$

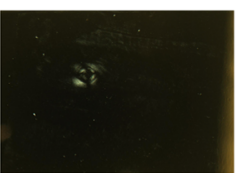

Crossed

polarizer

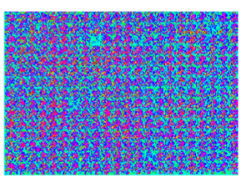

Diattenuation

axis orientation $\left(\theta_{D}\right)$

Fig. 5 Example data set showing all imaging parameters available from the holographic endomicroscope for a single mouse sample. Regions of interest are identified based on the legend given on the bottom right. (a) Bright-field, fluorescence, phase-contrast, and crossed-polarization microscopy of a sample containing a single lesion obtained using standard microscopy equipment. (b) Raw images from the holographic endomicroscope, including inferred polarimetric properties. (c) Expectation (i.e., spatial average) of the raw quantities. (d) Entropy of the raw quantities. Scale bar: $1 \mathrm{~mm}$.

entropy, most likely due to the high intersample variance. The statistically significant CNR suggests there may, nonetheless, be valuable information contained in the polarization data, but a more advanced classifier trained on a larger sample size would likely be required to evaluate the full potential of these techniques.

\section{Discussion}

Parallelized fiber TM characterization enables amplitude, quantitative phase, and resolved polarimetric imaging (termed here as "holographic endomicroscopy") to be performed on tissue phantoms and esophageal tissue samples. Quantitative phase and 

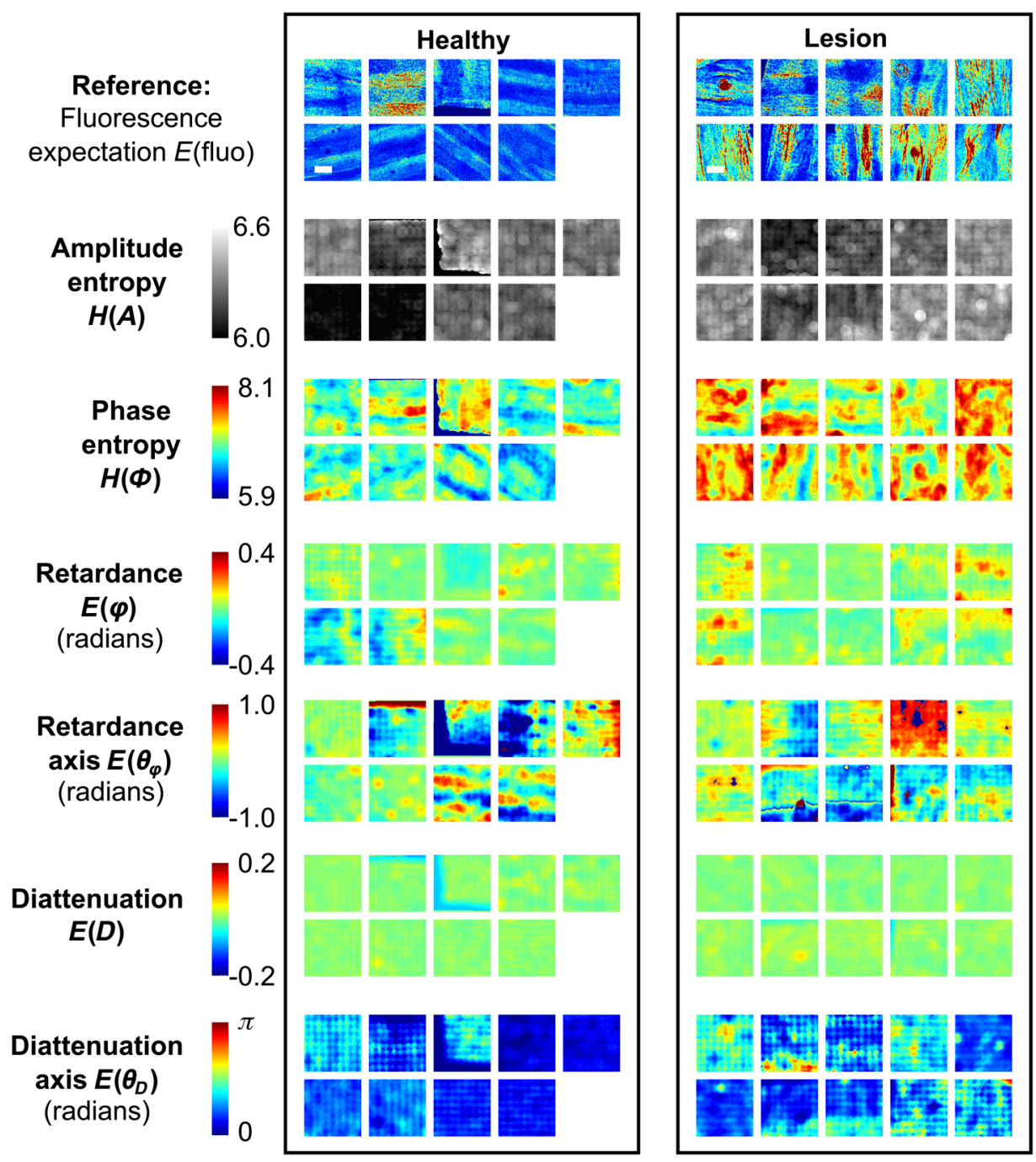

Fig. 6 Holographic endomicroscopy detects a range of early tumor lesions. Extracted regions of interest from full image data sets (see example in Fig. 5) showing sections of healthy tissue and lesions from all nine samples and five endomicroscope parameters. A range of focal and diffuse early tumor lesions can be observed in the reference fluorescence images. Scale bar $400 \mu \mathrm{m}$.

resolved polarimetric properties have previously shown promise for enhancing cancer detection in ex vivo and in vivo rigid endoscopy studies. ${ }^{3,51}$ Further, they are largely robust to variations in absolute intensity under varying measurement conditions. Retrieval of these properties through a flexible endoscope could therefore enhance contrast for premalignant and malignant changes during diagnostic endoscopy in the GI tract and lung.

To validate the potential of imaging quantitative phase and resolved polarimetric parameters in a holographic endoscope, we first successfully imaged tissue phantoms with biologically relevant amounts of scattering and birefringence, demonstrating a linear relationship between the known values and those measured through the endoscope. Next, we examined mouse esophageal tissue containing early abnormal lesions and compared our findings to healthy control tissue. We found that the spatial entropy of phase information, a result of surface scattering, provides significant contrast improvement relative to amplitude-only images and is comparable to fluorescent images using a nuclear stain typically used to identify these early lesions ex vivo. Similarly, a simple sum of three measured polarimetric parameters $\left(\theta_{D}, \theta_{\varphi}\right.$, and $\left.\varphi\right)$ provides significant contrast improvement compared to amplitude-only images and is comparable to the fluorescence reference. Phase entropy also provides the potential for very high specificity ( $>95 \%$ for $50 \%$ sensitivity), which would be an important consideration in a clinical setting for identifying low-risk cases (i.e., performing risk stratification and triage) in surveillance programs. Obtaining a label-free diagnosis equivalent to that provided by fluorescence staining avoids the need for extended procedure time and potential toxicities associated with in vivo dye application. ${ }^{5}$ Importantly, these results indicate that phase and polarimetric images may contain more relevant diagnostic information than amplitude-only images, demonstrating a key advance over what is possible using current commercial endoscopes. Nonetheless, examination of a greater diversity of lesion stages and tissue types, particularly from ex vivo human esophageal tissue, is required to further quantify the robustness of phase and polarimetric imaging for detecting early tumors in the esophagus. It would also be important to understand how such data can be interpreted by endoscopists. While our simple binary classifier showed promising performance in our ROC analysis, 
(a)

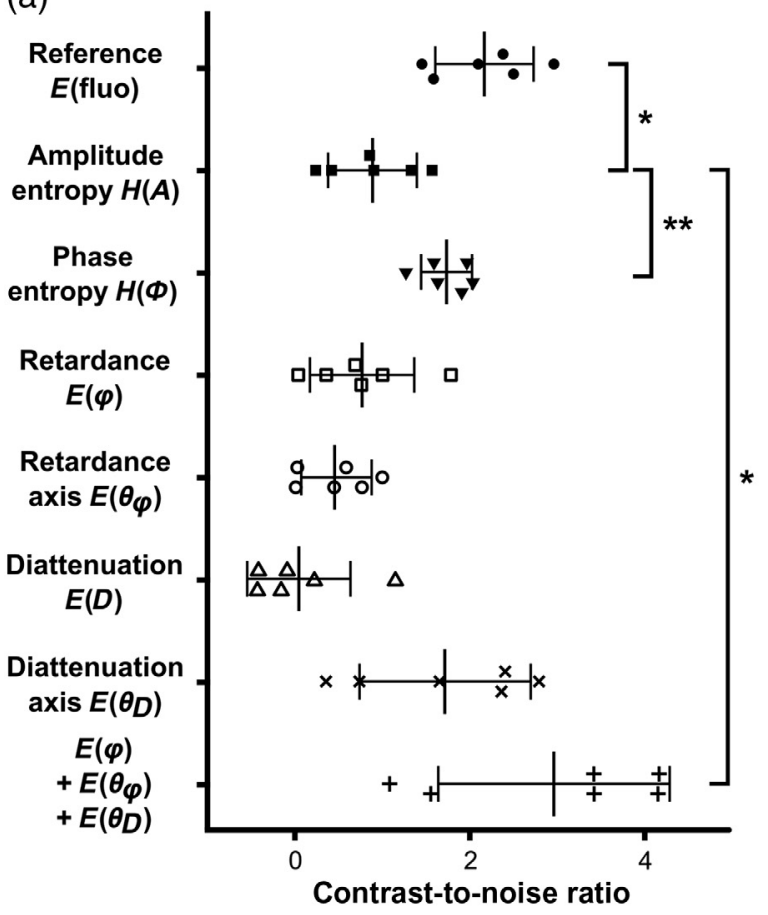

(b)

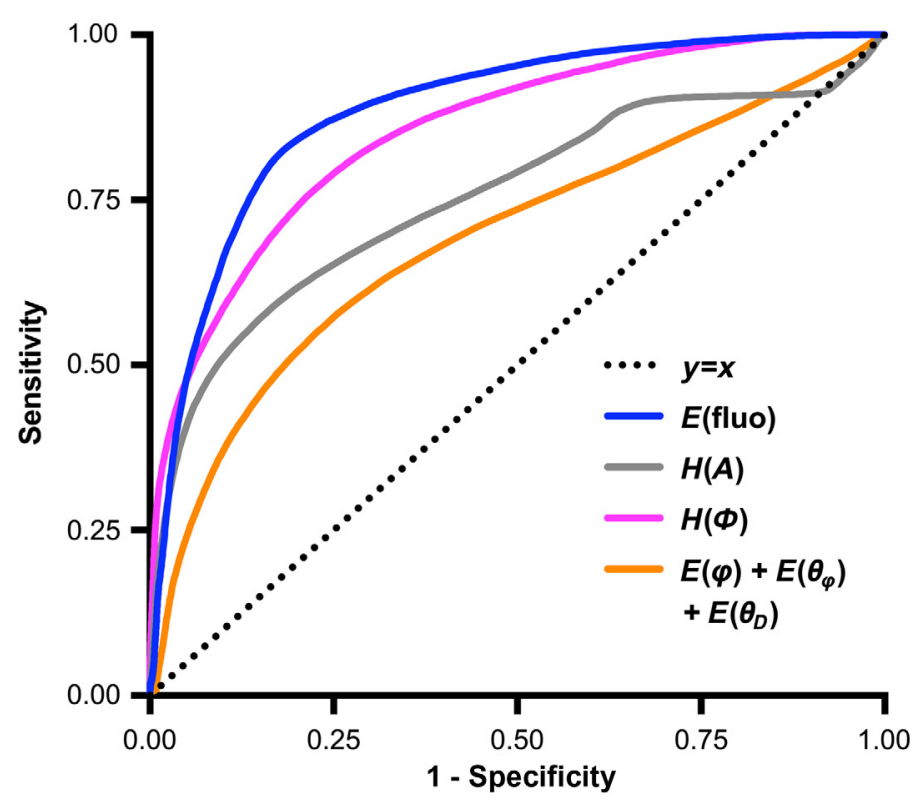

Fig. 7 Holographic endomicroscopy enables label-free identification of early lesions in the mouse esophagus. (a) CNR for the different parameters calculated independently for each of the six samples containing lesions. The phase entropy and sum of $\phi, \theta_{\phi}$, and $\theta_{D}$ polarization parameters produce contrast statistically comparable to fluorescence imaging, the gold standard reference modality $(p=0.148$ and $p=0.160$, respectively). Further, these two metrics produce significantly better contrast than amplitude entropy, the best available using conventional endomicroscopes ( $p=0.0014$ and $p=0.034$, respectively). Significance determined by paired two-tailed $t$-test, ${ }^{*} p<0.05$ and ${ }^{* *} p<0.01$. (b) Receiver operating characteristic curve illustrating performance of different parameters when a binary classifier with varying threshold is applied to discriminate between healthy and lesion tissues. Phase entropy significantly outperforms conventional amplitude imaging, but the large intersample variance of the combined polarization metric results in reduced performance with a simple binary threshold classifier.

with a larger dataset we could train a more advanced classifier that optimally combines all measured properties into a single contrast metric and facilitates identification of diseased areas.

Although we have demonstrated that retrieval of these additional optical parameters through an MCF endoscope enhances lesion visibility in the esophagus, the methodology used here is applied in transmission-mode imaging, so it is not directly translatable into clinical applications. We expect the heterogeneity observed in transmission mode to be preserved in reflection mode due the established correlation between forward and backward scattering. ${ }^{52}$ Further, spatial heterogeneity in reflectionmode imaging has been experimentally validated as method for identifying disordered tissue microstructure associated with tumors. ${ }^{41}$ Our findings provide an early feasibility study for the application of quantitative phase- and polarization-resolved imaging in a future in vivo setting and motivate further research to develop a reflection-mode holographic endoscopy.

Where access to the distal end of the MCF is not possible, the reported transmission-mode architecture would need to be reformatted to enable reflection-mode imaging. Oblique backscattering illumination can simulate transillumination in reflection, ${ }^{53}$ but in situ reflection-mode TM characterization is a significant challenge in the early stages of exploration. Compared to multimode fibres, MCFs have the significant advantage that without calibration they largely preserve the amplitude of light due to their pixellated structure, making registration and navigation in realistic clinical settings much easier. ${ }^{27,38}$ However, for accurate quantitative phase and polarization imaging, the TM must be characterized in reflection mode. Theoretical work by $\mathrm{Gu}$ et al. ${ }^{54}$ has proposed using light back-reflected from a known distal plate to dynamically update a prerecorded TM, but this requires a distal shutter and assumes a unitary TM, which is not typically the case for real fibers. ${ }^{37}$ Simulations indicate that a multilayered reflector stack placed on the distal end of a fiber could enable TM recovery in reflection mode for nonunitary fiber TMs and without a distal shutter by modulating wavelength. ${ }^{55}$ Further, experimental findings using a highly spaced MCF for two-photon imaging are also encouraging. ${ }^{56}$ These are promising indicators that experimental implementation of reflection-mode characterization is feasible. If successful, holographic endoscopy could enable retrieval of label-free quantitative phase- and polarization-resolved image metrics with the potential for application in early detection of esophageal tumors.

\section{Conclusion}

In summary, we demonstrated here detection of biologically relevant quantitative phase- and polarization-resolved properties from tissue-mimicking phantoms and early tumors in esophageal tissue through an MCF. 


\section{Disclosures}

The authors declare that there are no conflicts of interest related to this article.

\section{Acknowledgments}

This work was funded by Cancer Research UK (Nos. C47594/ A16267, C14303/A17197, and C47594/A21102); the European Union Seventh Framework Agreement No. FP7-PEOPLE-2013CIG-630729); and the Pump-Priming Awards from the Cancer Research UK Cambridge Centre, including dedicated funding from the Early Detection Program (No. A20976). We would like to thank Professor Sir Bruce Ponder and Professor Kevin Brindle for early input on our proof-of-concept studies. We would also like to thank summer students Callum Stevens, Sam Watcham, Khoa Pham, and Megan Wilson for their contributions to the tissue phantom characterization instruments that were used as reference gold standards in this work. Data associated with this publication is available at https://doi.org/ 10.17863/CAM.46316.

\section{References}

1. R. Anaparthy and P. Sharma, "Progression of Barrett oesophagus: role of endoscopic and histological predictors," Nat. Rev. Gastroenterol. Hepatol. 11(9), 525-534 (2014).

2. U. Navaneethan et al., "Radiofrequency ablation devices," VideoGIE 2, 252-259 (2017).

3. D. J. Waterhouse et al., "Emerging optical methods for endoscopic surveillance of Barrett's oesophagus," Lancet Gastroenterol. Hepatol. 3, 349-362 (2018).

4. N. Thosani et al., "ASGE Technology Committee systematic review and meta-analysis assessing the ASGE Preservation and Incorporation of Valuable Endoscopic Innovations thresholds for adopting real-time imaging-assisted endoscopic targeted biopsy during endoscopic surveillance of Barrett's esophagus," Gastrointest. Endosc. 83(4), 684-698.e7 (2016).

5. A. Doerr, "Imaging goes label-free," Nat. Methods 6(2), 116 (2009).

6. M. B. Sturm and T. D. Wang, "Emerging optical methods for surveillance of Barrett's oesophagus," Gut 64, 1816-1823 (2015).

7. D. J. Waterhouse et al., "A roadmap for the clinical implementation of optical-imaging biomarkers," Nat. Biomed. Eng. 3(5), 339-353 (2019).

8. J. Mannath et al., "Narrow band imaging for characterization of high grade dysplasia and specialized intestinal metaplasia in Barrett's esophagus: a meta-analysis," Endoscopy 42, 351-359 (2010).

9. M. Giacchino et al., "Clinical utility and interobserver agreement of autofluorescence imaging and magnification narrow-band imaging for the evaluation of Barrett's esophagus: a prospective tandem study," Gastrointest. Endosc. 77(5), 711-718 (2013).

10. H. Sparks et al., "A flexible wide-field FLIM endoscope utilising blue excitation light for label-free contrast of tissue," J. Biophotonics 8, 168-178 (2015).

11. J. F. de Boer, C. K. Hitzenberger, and Y. Yasuno, "Polarization sensitive optical coherence tomography a review [Invited]," Biomed. Opt. Express 8(3), 1838-1873 (2017).

12. G. Isenberg et al., "Accuracy of endoscopic optical coherence tomography in the detection of dysplasia in Barrett's esophagus: a prospective, double-blinded study," Gastrointest. Endosc. 62, 825-831 (2005).

13. N. G. Terry et al., "Detection of dysplasia in Barrett's esophagus with in vivo depth-resolved nuclear morphology measurements," Gastroenterology 140, 42-50 (2011).

14. L. Qiu et al., "Multispectral light scattering endoscopic imaging of esophageal precancer," Light: Sci. Appl. 7(4), 17174 (2018).

15. M. Ishigaki et al., "Diagnosis of early-stage esophageal cancer by Raman spectroscopy and chemometric techniques," Analyst 141(3), 1027-1033 (2016).

16. M. J. Gora et al., "Tethered capsule endomicroscopy enables less invasive imaging of gastrointestinal tract microstructure," Nat. Med. 19, 238-240 (2013).
17. M. Kim et al., "Label-free neuroimaging in vivo using synchronous angular scanning microscopy with single-scattering accumulation algorithm," Nat. Commun. 10(1), 1-9 (2019).

18. Z. Wang et al., "Tissue refractive index as marker of disease," J. Biomed. Opt. 16(11), 116017 (2011).

19. P. Ledwig and F. E. Robles, "Epi-mode tomographic quantitative phase imaging in thick scattering samples," Biomed. Opt. Express 10(7), 3605-3621 (2019).

20. Y. Wang et al., "Differentiating characteristic microstructural features of cancerous tissues using Mueller matrix microscope," Micron 79, 8-15 (2015).

21. G. Matz et al., "Chip-on-the-tip compact flexible endoscopic epifluorescence video-microscope for in-vivo imaging in medicine and biomedical research," Biomed. Opt. Express 8(7), 3329-3342 (2017).

22. K. K. Ghosh et al., "Miniaturized integration of a fluorescence microscope," Nat. Methods 8(10), 871-878 (2011).

23. C. Hu et al., "Endoscopic diffraction phase microscopy," Opt. Lett. 43(14), 3373 (2018).

24. R. Horisaki et al., "Single-shot phase imaging with a coded aperture," Opt. Lett. 39, 6466 (2014).

25. H. Luo et al., "Compact and miniature snapshot imaging polarimeter," Appl. Opt. 47, 4413 (2008).

26. S. M. Popoff et al., "Measuring the transmission matrix in optics: an approach to the study and control of light propagation in disordered media," Phys. Rev. Lett. 104, 100601 (2010).

27. G. S. D. Gordon et al., "Full-field quantitative phase and polarisationresolved imaging through an optical fibre bundle," Opt. Express 27, 23929-23947 (2019).

28. R. Simon, "The connection between Mueller and Jones matrices of polarization optics," Opt. Commun. 42, 293-297 (1982).

29. S. Turtaev et al., "Exploiting digital micromirror device for holographic micro-endoscopy," Proc. SPIE 10932, 1093203 (2019).

30. Z. Zhang et al., "A survey of sparse representation algorithms and applications," IEEE Access 3, 490-530 (2015).

31. E. van den Berg and M. P. Friedlander, "SPGL1: a solver for large-scale sparse reconstruction," 2007, https://www.cs.ubc.ca/ mpf/spg11/.

32. L. Allen and M. Oxley, "Phase retrieval from series of images obtained by defocus variation," Opt. Commun. 199, 65-75 (2001).

33. M. Gataric et al., "Reconstruction of optical vector-fields with applications in endoscopic imaging," IEEE Trans. Med. Imaging 38, 955-967 (2019).

34. M. Tanter, J.-L. Thomas, and M. Fink, "Time reversal and the inverse filter," J. Acoust. Soc. Am. 108(1), 223-234 (2000).

35. S. Popoff et al., "Image transmission through an opaque material," Nat. Commun. 1, 1-5 (2010).

36. S. Rotter and S. Gigan, "Light fields in complex media: mesoscopic scattering meets wave control," Rev. Mod. Phys. 89(1) (2017).

37. J. Carpenter, B. Eggleton, and J. Schröder, " $110 \times 110$ optical mode transfer matrix inversion," Opt. Express 22(1), 96-101 (2014).

38. G. S. D. Gordon et al., "Coherent imaging through multicore fibres with applications in endoscopy," J. Lightwave Technol. 37, 5733-5745 (2019).

39. F. Albregtsen, "Statistical texture measures computed from gray level coocurrence matrices," Technical Report, University of Oslo, Oslo, Norway (2008).

40. A. Rényi, "On measures of entropy and information," in Proc. Fourth Berkeley Symp. Math. Stat. Prob. Vol. 1: Contrib. Theory of Stat., University of California Press, Berkeley, California, pp. 547-561 (1961).

41. D. M. McClatchy et al., "Wide-field quantitative imaging of tissue microstructure using sub-diffuse spatial frequency domain imaging," Optica 3(6), 613 (2016).

42. K. Yogesan et al., "Entropy-based texture analysis of chromatin structure in advanced prostate cancer," Cytometry 24, 268-276 (1996).

43. P. F. F. de Arruda et al., "Quantification of fractal dimension and Shannon's entropy in histological diagnosis of prostate cancer," BMC Clin. Pathol. 13, 6 (2013).

44. B. Ganeshan et al., "Hepatic enhancement in colorectal cancer. Texture analysis correlates with hepatic hemodynamics and patient survival," Acad. Radiol. 14(12), 1520-1530 (2007).

45. J. Joseph et al., "Evaluation of precision in optoacoustic tomography for preclinical imaging in living subjects," J. Nucl. Med. 58(5), 807-814 (2017). 
46. J. W. Pickering et al., "Double-integrating-sphere system for measuring the optical properties of tissue," Appl. Opt. 32(4), 399 (1993).

47. S. A. Prahl, M. J. C. van Gemert, and A. J. Welch, "Determining the optical properties of turbid media by using the adding-doubling method," Appl. Opt. 32(4), 559 (1993).

48. M. P. Alcolea et al., "Differentiation imbalance in single oesophageal progenitor cells causes clonal immortalization and field change," Nat. Cell Biol. 16, 615-622 (2014).

49. N. Ghosh, "Tissue polarimetry: concepts, challenges, applications, and outlook," J. Biomed. Opt. 16(11), 110801 (2011).

50. Y. Choi et al., "Scanner-free and wide-field endoscopic imaging by using a single multimode optical fiber," Phys. Rev. Lett. 109, 203901 (2012).

51. J. Qi and D. S. Elson, "A high definition Mueller polarimetric endoscope for tissue characterisation," Sci. Rep. 6, 25953 (2016).

52. I. Starshynov et al., "Non-Gaussian correlations between reflected and transmitted intensity patterns emerging from opaque disordered media," Phys. Rev. X 8(2), 21041 (2018).

53. T. N. Ford, K. K. Chu, and J. Mertz, "Phase-gradient microscopy in thick tissue with oblique back-illumination," Nat. Methods 9(12), 1195-1197 (2012).
54. R. Y. Gu, R. N. Mahalati, and J. M. Kahn, "Design of flexible multimode fiber endoscope," Opt. Express 23(21), 26905-26918 (2015).

55. G. S. D. Gordon et al., "Characterising optical fibre transmission matrices using metasurface reflector stacks for lensless imaging without distal access," arXiv:1904.02644 (2019).

56. S. C. Warren et al., "Adaptive multiphoton endomicroscopy through a dynamically deformed multicore optical fiber using proximal detection," Opt. Express 24(19), 21474-21484 (2016).

George S. D. Gordon is an assistant professor in the Department of Electrical and Electronic Engineering at the University of Nottingham, United Kingdom. Prior to that he was a research fellow at the University of Cambridge, United Kingdom. His research is on optics and photonics for applications in medical imaging.

Biographies of the other authors are not available. 